\title{
Eliciting and Modeling Business Process Stories
}

\author{
David Simões $\cdot$ Pedro Antunes $\cdot$ Luis Carriço
}

Received: 17 June 2015/Accepted: 19 May 2016/Published online: 11 May 2017

(C) Springer Fachmedien Wiesbaden 2017

\begin{abstract}
Most business process modeling tools used today encourage the rendition of lean, prescribed and clearly coordinated activities, which often clash with far more intricate business realities. This paper evaluates an alternative approach that adopts concepts from storytelling and sense-making theories to elicit process stories. The viability of this approach is tested in a case study involving sixteen individuals from the same organization who tell their personal views about a business process, thus gathering a total of twenty process stories. The obtained results suggest that process stories may increase the meaningfulness, contextualization and overall richness of process models.
\end{abstract}

Keywords Business process modeling · Organizational storytelling $\cdot$ Process stories $\cdot$ Modeling tools

\section{Introduction}

A defining characteristic of Business Process Management (BPM) is that it requires accurate representations of

Accepted after two revisions by Prof. Dr. Becker.

Electronic supplementary material The online version of this article (doi:10.1007/s12599-017-0475-3) contains supplementary material, which is available to authorized users.

Prof. D. Simões $(\bowtie) \cdot$ Prof. L. Carriço

Faculty of Sciences, University of Lisbon, Campo Grande,

1749-016 Lisbon, Portugal

e-mail: david.simoes@gmail.com

Prof. P. Antunes

School of Information Management, Victoria University of Wellington, PO Box 600, Wellington 6140, New Zealand operational and managerial activities in order to enact it in process-aware technology such as workflow, document management, enterprise resource planning, and supply chain management. Several modeling techniques and associated languages have been adopted to represent business processes, including Flowcharting (Lakin et al. 1996), Petri Nets (Schnupp and Muchnick 1992), Role Activity Diagrams (Holt et al. 1983), IDEF (Mayer et al. 1995), Event Driven Process Chain (EPC) (Scheer et al. 2005), UML Activity Diagrams (Object Management Group 2011), YAWL (van der Aalst and ter Hofstede 2005), and the Business Process Model and Notation (BPMN) (Object Management Group 2013). The research literature provides several comparisons of the different techniques and languages, showing how they address a wide range of business needs, such as redesigning the way organizations do their business, and also a wide range of technical needs, like information systems integration and automation (Hommes and van Reijswoud 2000; Giaglis 2001; Aguilar-Saven 2004; List and Korherr 2006).

However, several weaknesses have been identified in the existing modeling techniques and languages (Russell et al. 2006; Wohed et al. 2006). In particular, many researchers have expressed concerns with the lack of flexibility in process models, which often lead to systemic failures caused by unexpected events and conditions as well as ad hoc business needs (Pesic 2008; Antunes and Mourão 2011; Jonnavithula et al. 2015). Also related to flexibility, some researchers have discussed the dilemmas of control, i.e., how control should fluctuate between humans and machines to adapt to work constraints (Cabitza and Simone 2013). Moreover, several studies mention that often process models are regarded as overly prescriptive, when in reality organizations use them as information resources, useful but competing with other equally useful resources 
(Lindsay et al. 2003; Recker et al. 2006; Krogstie 2007; Riemer et al. 2014).

The lack of consideration for contextual knowledge in process modeling and execution has also been pointed out as an important limitation of current process modeling approaches, languages, tools, and systems (Caetano et al. 2005; Rosemann et al. 2008; Brossard et al. 2011; da Cunha Mattos et al. 2014). Combining work procedures with additional knowledge about constraints, deviations, operative scenarios, and past positive and negative outcomes, which may influence the specific details about how a particular process may unfold in a particular context, may help to reduce gaps between model and reality (Antunes et al. 2013).

In our research (Antunes et al. 2013; Simões et al. 2016), we have adopted the perspective that contextual knowledge should be an integral part of business process management. Our standpoint is informed by organizational theory, in particular the sense-making (Weick 1993, 1995) and organizational storytelling (Denning 2000, 2006) theories, which suggest that decisions made by people in organizational settings are influenced by perception, recognition, past experience, and also purposeful action and feedback. In particular, the storytelling stance suggests that process models should extend their representational abilities beyond the strict representation of activities, conditions and flows, towards what we designate by process stories. Process stories may hold contextually richer, diversified information about the activities being modeled. For instance, process stories may combine typical with atypical situations in the work routine, identifying expected and unexpected events that have occurred in the past, mentioning the considerations and decisions that were then made, while highlighting the organizational culture, rituals and practices affecting them.

In our prior research, we developed a tool supporting the elicitation and modeling of process stories (Antunes et al. 2013). We proposed a meta-model that incorporates elements from storytelling theory with elements typically found in traditional process modeling languages and tools. Furthermore, we integrated the concept of process story with a visual narrative scheme inspired by comic strips, using textual/visual elements and rich metaphors to express contextual knowledge (Short and Reeves 2009). In particular, the tool helps externalizing organizational knowledge related to business processes in a visual way, using generic pictures of business situations, like having a meeting or working on a document, in combination with other textual and visual elements such as dialogue lines, which help characterizing places, activities, actors, business objects, thoughts, decisions, and other intervening factors. The use of sketches and storyboards are particularly well suited for storytelling-based process modeling, as they (1) integrate graphical shapes and textual elements leading to more effective design representations (Recker et al. 2010), (2) abstract away detail and the sense of finality that would be present in a photograph to trigger creativity, imagination and immersion in the story (Williams and Alspaugh 2008), (3) ease user identification with the characters - "the stylized character is, [...] an empty shell that we inhabit" (McCloud 1993), (4) are intuitive and enhance memory retention (Gershon and Page 2001).

Even though the tool has already been informally tested and provided good indications about the viability of the approach (Antunes et al. 2013; Simões et al. 2016) we lacked insights from a more thorough study demonstrating the utility of process stories. The current study aims to cover this gap. We are specifically interested in answering the following research questions:

RQ1 Is it feasible for stakeholders to elicit meaningful business processes without the participation of expert modelers?

RQ2 Is business process context extractable from process stories?

RQ3 How do process stories improve the modeling of business processes?

The paper is structured as follows. The next section presents background information regarding related work, the role of process modeling, and prior experiments using our modeling approach. In Sect. 3 we discuss the case study design including objectives, research questions, data collection procedures, and analysis. Section 4 presents the results from the case study. Lastly, we present some conclusions and future work directions.

\section{Background}

\subsection{Related Work}

Business process automation, integrating execution, analysis and reengineering activities, can arguably be considered the ultimate goal of business process modeling, contributing to deeper understanding and systematization of the network of activities, prescribed or otherwise, that compose a business (Kock et al. 2009; Dumas et al. 2013). The utility of process modeling is not however limited to automation. A common goal for process modeling efforts is to increase knowledge and shared awareness within the organization (Bandara et al. 2005).

Fleischmann et al. (2012) recognized that the transference of knowledge between stakeholders and expert modelers is often flawed, leading to losses of information and inconsistencies reflecting actual work procedures. Their subject-oriented approach (S-BPM) puts stakeholders at the 
center of attention. During S-BPM analysis, natural language and its structuring elements are used to identify activities and the intervening actors and business objects. Our approach is consistent with the S-BPM perspective. It too is subject-oriented and regards process context as important to modeling. However, while S-BPM is mainly centered on control flows (Fleischmann et al. 2013), we are mostly focused on the more diverse information brought by process stories. Besides, we elicit process stories directly from the stakeholders, without mediation from modeling experts, thus avoiding potential gaps in knowledge transference and translation to modeling languages. We argue that process modeling should ideally be left to the stakeholders, so they can describe business processes from diverse, complementary perspectives.

Harman et al. (2015) presented a role-playing approach to process elicitation where stakeholders navigate a virtual world and perform process-related tasks. The method aims to assist in memory recollection. Common to our approach, process information is gathered without support from expert modelers. However, while this role-playing approach is centered on formally modeling process activities, our approach is focused on capturing the context of such activities. By eliciting context, we aim to gain a better understanding of business processes, especially beyond the "happy path" scenario.

Nardella et al. (2014) adopted a storyboarding technique to create $3 \mathrm{D}$ visualizations of process models, with the goal of improving communication between expert modelers and stakeholders with no modeling experience. In our approach, storyboarding is used not only to show process information to stakeholders, but also and most importantly, to help stakeholders in building the process stories themselves, and in sharing and discussing these stories to increase process knowledge and awareness.

Mayer (1989) work on cognitive theory and learning demonstrated that models improve conceptual retention while deemphasizing verbal retention, and improve problem-solving transfer skills. The learning process is dependent not only on the contents being transmitted, but also on the way it is presented and the individual characteristics of the audience. Based on Mayer's research, Recker and Dreiling (2007) investigated whether familiarity with a particular modeling language would play a role in model understanding, but found no significant differences in performance by modelers specializing in either EPC or BPMN when interpreting models expressed in each language. Figl and Recker (2014) found an overall tendency among business users of process models to prefer diagrammatic representations over text (both structured and free-form), and to prefer iconic representations when task settings relate to understanding and communicating process models. Mendling et al. (2010) suggested using icons to complement textual labels representing activities, with the aim of easing model understanding. In our approach, we use graphical illustrations to represent business situations as part of process stories. However, we refer to business situation in a wider scope. It may describe one or more process activities, or portray contextual information about the process. We use these illustrations to complement textual descriptions (the narrative) with the purpose of improving model comprehension and user engagement in the story.

Ottensooser et al. (2012) studied the comprehensibility of design notations, comparing the BPMN notation with textual notations (in the form of written business process descriptions). The study shows that both expert modelers and stakeholders benefit from textual descriptions of business processes followed by the corresponding BPMN diagrams. Moreover, for stakeholders, BPMN diagrams taken alone did not significantly increase process understanding beyond their background knowledge of the domain. These results show that diagrammatic representations such as BPMN are needed to convey precise semantics but complementary, more familiar, text-based representations can also play a valuable role in model comprehension from the stakeholders. The lack of intuitiveness of diagrammatic representations and the complementary role of text-based representations has been underlined in recent research (Chakraborty et al. 2010; Dumas et al. 2013; van der Aa et al. 2015a). In this context, efforts have been devised to approximate the two approaches, either by generating natural language texts from process models (Leopold et al. 2014), or by automatically detecting inconsistencies between diagrammatic and textbased descriptions of the same business processes (van der Aa et al. 2015b).

\subsection{Proposed Method and Modeling Tool}

In our approach, we deemphasize the role of expert modelers and rely on the stakeholders to do the elicitation and modeling work. The central aspect of our method is to get stakeholders to recollect their daily work activities and to develop business stories describing actual procedures in contextualized settings. According to van der Aalst (2013) classification, our method handles descriptive process models. However, these descriptions avoid the typical formalisms used by most process modeling tools like AND-splits, AND-joins, XOR-splits, etc. (van der Aalst et al. 2003). Instead, inspired by organizational storytelling theory, models are organized around scenes. Specifically, stakeholders are invited to create stories by visually assembling a sequence of scenes portraying business situations and providing context by enriching scenes with dialogue and narrative content. 
Fig. 1 Storytelling model (some classes and attributes are omitted for clarity)

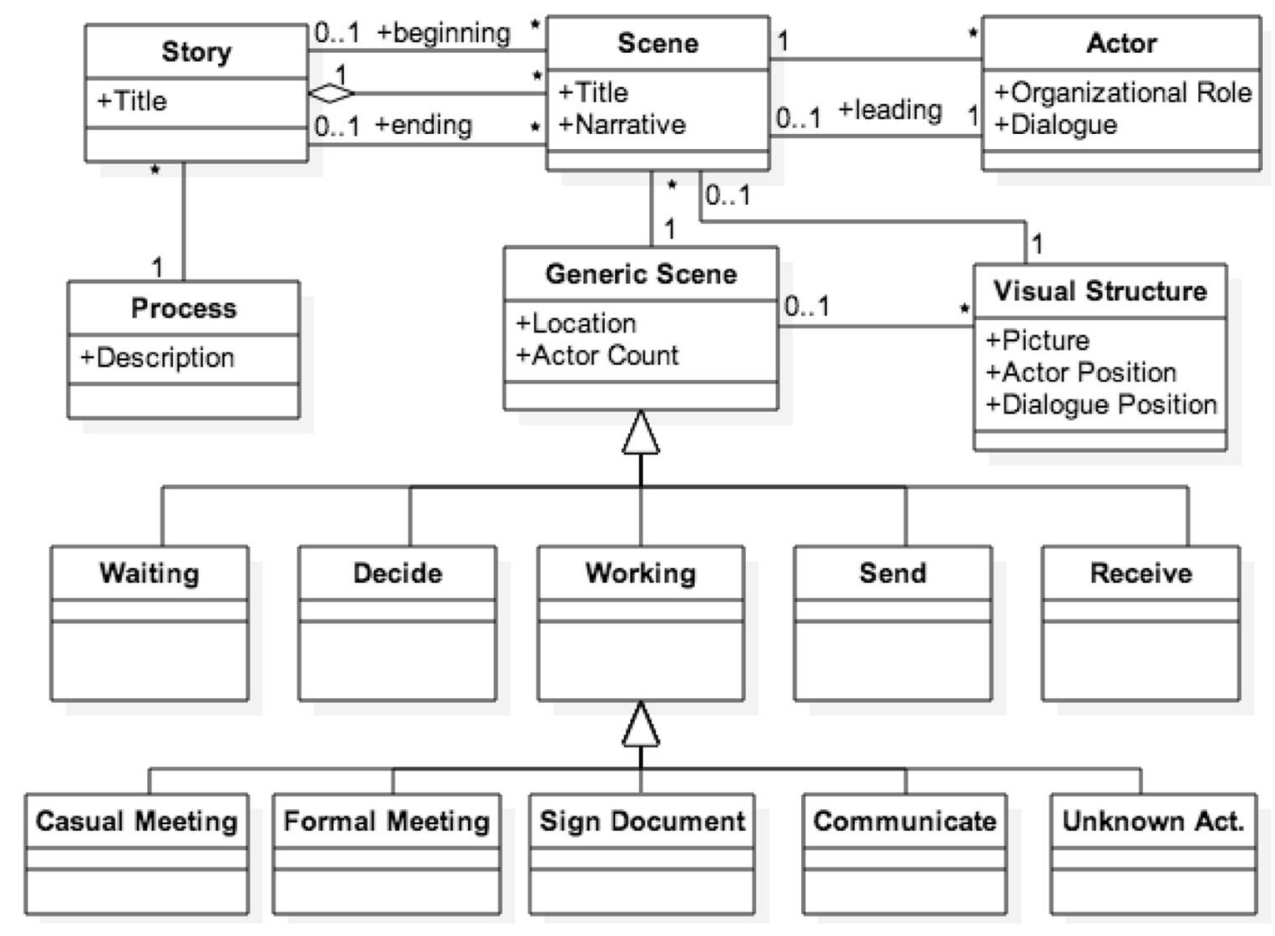

A scene is a unit of knowledge that conveys contextual information about business activities. The proposed storytelling model, represented in Fig. 1, defines the following attributes of a scene. It has a specific setting, indicating the physical location where activities occur, e.g., an office or department; and also indicating the particular situation or setting, like communicating, signing a document and making a business decision. A scene may involve several actors, either persons or machines that directly intervene in the scene. Physical and virtual artefacts, like tools and documents, may also be involved in scenes. Several events and actions may be reported in a scene; they drive the storyline in terms of purposeful action. Finally, dialogue lines can be added to actors to better express their involvement in the scene, e.g. making decisions and externalizing knowledge.

A scene incorporates a cartoon-like graphical illustration and scene-specific metadata providing an abstract structure for the situation being depicted. The stakeholders are not expected to sketch these scenes. Instead, they select them from a library of over 100 generic, reusable scenes covering most common business settings, which are then configured to express a particular business process. For instance, the library contains a generic "waiting scene" illustration, which includes scene-specific metadata expressing the notion of time. The combination of time with the other metadata elements present in the waiting scene, in particular actors and events, allow expressing how long the actors are expected to wait and what actions will be executed when either the expected event occurs or the time limit is reached. There are additional types of generic scenes depicting common business situations and actions, such as making decisions, sending or receiving business artefacts, taking formal or casual meetings, signing documents, and communicating information (see Fig. 1). These major types of scenes and related metadata are implicitly mapped to different process constructs such as activities, conditions and events. Scenes are contextualized in the story by identifying the business artefacts present in the scene, mapping actors to organizational roles, and adding dialogue lines and narrative remarks. The story emerges as a sequence of scenes describing a process scenario that has occurred. A representative set of stories covering different scenarios and diverse points of view forms the process model.

We stress that scenes should be seen as templates and are meant to be reusable, and this is why we resort to drawings instead of images from the workplace in question. Of course, scenes explore the visual properties of graphical illustrations. They depict business situations in evocative ways and have to be interpreted, i.e. business people are expected to recognize and associate a scene to a particular business situation, activity, or rule. To develop a process story, users pick generic scenes from the library, organize them in sequential order, and then customize each scene according to the metadata outlined above. The outcome is a process story resembling a comic strip, where the plot (outlining a specific business process scenario) is unveiled 
and explained throughout the scenes using attributes like settings, actors, dialogues, events, actions, artefacts, and time stamps.

Our modeling tool offers a web-based authoring environment for visual composition and sharing of process stories (Borges and Pino 1999; Perret et al. 2004; Santoro et al. 2010). Figure 2 shows the tools' user-interface for composing a scene according to our approach. The user starts by selecting the type of situation from a dynamic list. Based on the selected type of situation, the tool asks for more specific information, which depends on the scenespecific metadata. In Fig. 2, the selected situation was "Send"; hence the tool asked details about what had been sent (e.g. an artefact) and who was involved in the exchange. The user could complete the scene by adding dialogue lines.

Upon saving a scene, the tool picks a picture from the database portraying the situation and presents it to the user, who is left free to focus her attention on developing the process story. Often the database has several alternative pictures for the same situation and the user is allowed to choose one of them. The complete process story is then viewable either in a booklet printable form or interactively, moving forth and back the list of scenes.

\subsection{Prior Evaluation Studies with the Tool}

In a previous article (Antunes et al. 2013) we report a preliminary evaluation study of the modeling tool and process stories. The study suggested that the stakeholder- centric elicitation of business processes through process stories was feasible however hindered by several constraints imposed by the tool's limited usability.

Later on (Simões et al. 2016), we reported a case study with a revised tool where a small team was involved in the analysis and design of an improved business process that was critical to them. All team members were involved in generating process stories, including the team leader. Then the leader used the individual process stories to suggest a converged process model, which was finally discussed and acquiesced by the team.

The results from these studies showed that meaningful business stories could indeed be elicited and modeled by the stakeholders without mediation from modeling experts. Nevertheless, even though there were some positive indications about the method of process modeling through storytelling, we found out that stories lacked or were poor on the depiction of unexpected situations, personal views, and situated reasoning, which are the foundations of process contextualization. In the abovementioned study, the team scarcely used dialogue and was heavily reliant on traditional activity flows, when we were expecting rich stories full of exceptional events and insights about what happens in reality. That is, although the method and tool were found to be viable for business process modeling, our main hypothesis that process stories could enable process contextualization was not validated.

Two factors have emerged as possible reasons for these less desirable results. The first aspect that we noted was that, by chance, the team was specifically seeking to create
Fig. 2 The modeling tool's user-interface. 1 The title of the scene; 2 Narrative description of what happens in the scene; 3 Actor identification and mappings to organizational roles; 4 Scene dialogue; 5 Flow to the next scene; 6 Control over picture selection and dialogue positioning; 7 Visual representation and navigation between scenes

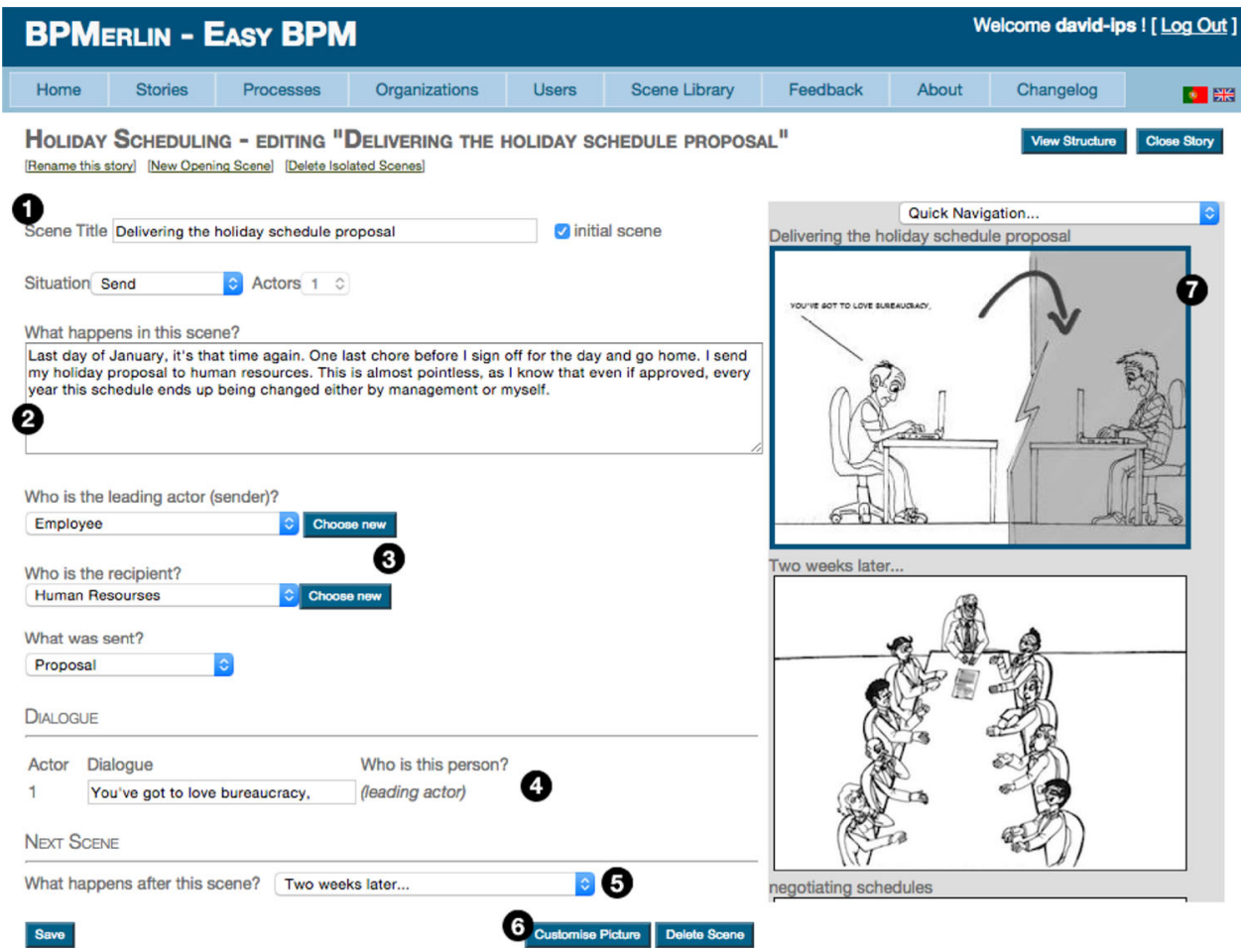


a completely new business process, and thus their stories reflected more how they thought the process ought to be rather than what actually was happening in day-to-day situations. As such, the stories were prescriptive as opposed to contextualized and episodic. Moreover, the fact that the participants were skilled IT practitioners, all with backgrounds in software engineering, may have also affected the way they conceptualized the business process. In particular, the participants revealed an inclination for abstracting processes using typical computer-science paradigms (e.g. decomposition, separation of concerns, iteration), which favor the traditional representation of activities, conditions and flows. The study reported in this paper addresses these concerns.

\section{Case Study Design}

We followed case study research methodology from Benbasat et al. (1987) and guidelines from Runeson and Höst (2009). Throughout this section we address site selection, unit of analysis determination, case study protocol, ethical considerations, data collection, triangulation, metrics, data analysis, and validity.

We considered three major requirements for the case study design that drove the choice of the target organization and selected business process. These are described in the following paragraphs.

The first requirement concerned process complexity. We needed a minimum level of complexity for the process being analyzed to avoid investigating a near-trivial problem and to maximize the potential for grabbing contextually rich business stories. As such, we needed a process with multiple lingering steps, several task interdependencies, and also requiring problem solving. Most importantly, the process should summon the participation of various people across different business units. The intervention of multiple stakeholders from different units is usually a good source of complexity and enables the emergence of different, complementary and often conflicting, personal views, which again are recognizable sources of complexity.

Another requirement we had to consider concerned modeling bias. The study participants should not possess any specific expertise in computer science that would bias the modeling task, other than basic computer literacy and experience with worksheet software. The main reason was that in the previous study we found out that process modeling may have been affected by the participants' background in that field, which may have contributed to an emphasis on abstraction and decomposition.

The third requirement was that the target organization should be looking to model existing process knowledge.
This again contrasts with the prior study where the participants were seeking to develop a completely new process. As already mentioned, the focus on new process knowledge seems to emphasize prescriptive rather than descriptive process stories. Hence, for the current study, we were looking for study participants with an interest in documenting an existing process and no immediate plans for process reengineering or enactment.

The selected organization was the Polytechnic Institute of Setubal, hereinafter referred to as IPS, and the unit of analysis was the process by which a student requests and receives a course certificate or diploma upon completion of a study cycle. This process will be named the "diploma process". The study's participants were administrative staff belonging to the IPS Academic Division (AD), which is the process owner.

The AD is organized in three separate working units. The Front-Office establishes the interface between the AD and the students. Its responsibilities include receiving student requests, preliminary checking several requirements, communicating with administrative personnel, and following-up on processes by contacting students whenever necessary. The Treasury Office handles all payments made to the $\mathrm{AD}$, including tuitions and request emoluments. The Back-Office unit processes the requests. This comprises validating all requirements, contacting staff from other organizational units to track missing information and to comply with mandatory rules, emitting legal documents, and managing electronic records.

This study had the participation of all AD's staff. This included the head of the AD (designated Chief of Division), and fifteen workers allocated to the three units outlined above: five persons in the Front-Office, three persons in the Treasury Office, and seven persons in the BackOffice. Among the latter group, one person designated as Back-Office Coordinator is responsible for the operations at the $\mathrm{AD}$ and reports directly to the Chief of Division. Excluding the Chief of Division, all participants used the modeling tool.

The participants used the tool as an authoring environment for telling business stories. Other than a brief presentation session, we avoided direct contact with the participants, and used second-degree data collection techniques to obtain the user stories and associated data (Lethbridge et al. 2005). The participants in this study created their stories autonomously and data was collected at a later time from both the tool's print outs and raw usage logs. We resorted to a goal-based metric technique similar to the methodology proposed by Basili and Weiss (1984) to analyze the data. The specific measurement goals were aligned with the research questions established for this study. We then specified several questions and metrics necessary to evaluate the goals. The research questions, 
Table 1 Goal-based measurements

\begin{tabular}{|c|c|c|c|c|c|}
\hline RQ & Goal & Questions & Metric & $\begin{array}{l}\text { Type of } \\
\text { data }\end{array}$ & $\begin{array}{l}\text { Measurement } \\
\text { scale }\end{array}$ \\
\hline \multirow[t]{6}{*}{1} & \multirow{6}{*}{$\begin{array}{l}\text { Evaluate } \\
\text { meaningfulness }\end{array}$} & \multirow{6}{*}{$\begin{array}{l}\text { Did the subjects create detailed } \\
\text { stories? } \\
\text { Could workflows be derived } \\
\text { from user stories? }\end{array}$} & Number of scenes & Quantitative & Ratio \\
\hline & & & $\begin{array}{l}\text { Scene density (no. of scenes vs. no. of } \\
\text { process activities) }\end{array}$ & Quantitative & Ratio \\
\hline & & & Word count (narrative ${ }^{\mathrm{a}}$ ) & Quantitative & Ratio \\
\hline & & & Word count (dialogue ${ }^{\mathrm{b}}$ ) & Quantitative & Ratio \\
\hline & & & Structural complexity ${ }^{\mathrm{c}}$ & Qualitative & $\begin{array}{l}\text { Ordinal (low/ } \\
\text { medium/high) }\end{array}$ \\
\hline & & & $\begin{array}{l}\text { Story verifies process validity } \\
\text { requirements (see Antunes et al. 2013) }\end{array}$ & Qualitative & $\begin{array}{l}\text { Nominal (yes/ } \\
\text { no) }\end{array}$ \\
\hline \multirow[t]{3}{*}{2} & \multirow[t]{3}{*}{$\begin{array}{l}\text { Evaluate } \\
\text { contextualization }\end{array}$} & $\begin{array}{l}\text { Did participants express } \\
\text { contextual judgement? }\end{array}$ & $\begin{array}{l}\text { Presence of personal views in the } \\
\text { dialogue/narrative }\end{array}$ & Qualitative & $\begin{array}{l}\text { Nominal (yes/ } \\
\text { no) }\end{array}$ \\
\hline & & $\begin{array}{l}\text { Were unexpected situations } \\
\text { depicted? }\end{array}$ & Depiction of unexpected situations & Qualitative & $\begin{array}{l}\text { Nominal (yes/ } \\
\text { no) }\end{array}$ \\
\hline & & $\begin{array}{l}\text { Was contextualized knowledge } \\
\text { applied? }\end{array}$ & Detachment from prescribed procedure & Qualitative & $\begin{array}{l}\text { Nominal (yes/ } \\
\text { no) }\end{array}$ \\
\hline \multirow[t]{4}{*}{3} & \multirow{4}{*}{$\begin{array}{l}\text { Evaluate model } \\
\text { richness and adequacy }\end{array}$} & \multirow{2}{*}{$\begin{array}{l}\text { How detailed were the } \\
\text { activities? }\end{array}$} & Number of activities & Quantitative & Ratio \\
\hline & & & Number of organizational roles & Quantitative & Ratio \\
\hline & & How many distinct scenarios & Number of decision steps & Quantitative & Ratio \\
\hline & & $\begin{array}{l}\text { Does the model verify } \\
\text { organizational requirements? }\end{array}$ & Model reflects intended procedure & Qualitative & $\begin{array}{l}\text { Nominal (yes/ } \\
\text { no) }\end{array}$ \\
\hline
\end{tabular}

${ }^{a}$ We define narrative as the textual report made by the narrator of the story. In the modelling tool, narrative is registered in the text field labelled 2 in Fig. 2

${ }^{\mathrm{b}}$ We define dialogue as the direct discourse depicting individual thoughts or remarks, or spoken interactions amongst actors in the story. In the modelling tool, dialogue is registered in the text field labelled 4 in Fig. 2 (one text field per actor in the scene), and appears in the visual frame representing the scene

${ }^{c}$ Qualitative assessment of structural complexity is based on metrics from RQ3

goals, questions and metrics involved in the data analysis are shown in Table 1.

To ensure validity, the case study design was developed with knowledge and feedback from the Chief of Division. Furthermore, the study also received explicit agreement and commitment from all participants. This ensured that the study was aligned with the IPS goals and the target process and activities were considered adequate and relevant both for our research and the target organization.

The participants were briefed on the study protocol, including timings and data acquisition procedures. Ease of communication and alignment between researchers and participants' views on the case study were also favored by selecting a familiar target organization, i.e. the researchers had in-depth knowledge about the organizational culture and the participants were also aware of the constraints imposed by research practice.

Reliability and internal threats to validity were addressed as follows. We ensured that subjects understood the tasks they were about to perform by running a preliminary session with all participants where tasks were explained and the tool was demonstrated (more on that later). By not intervening during the modeling activities, we also ensured that the researchers did not influence the participants during the creation of their stories. Factors that could affect causal relations were also isolated, such as whether the participants were correctly using the tool, and the tool's adequacy for authoring business process stories.

We used different forms of triangulation to increase the quality of data analysis. We gathered data from multiple sources, namely from the tools' print outs (storyboards and structure diagrams), from raw system logs, and from the participants' feedback. We took both quantitative and qualitative measurements whenever possible, and data was analyzed independently by two researchers.

Ethical considerations included informed consent from all participants, who were briefed on the associated risks, privacy issues, and possible benefits brought by the study to the organization. As an added measure for ensuring a reasonable level of confidentiality, we configured the tool to optionally submit anonymous stories. This procedure was deemed appropriate considering the sensitive content potentially held in stories, such as diverting from established rules or even critiquing accepted practices.

Data gathering was conducted in three phases (see Fig. 3). The preliminary phase consisted on a single, 
Fig. 3 Field procedures

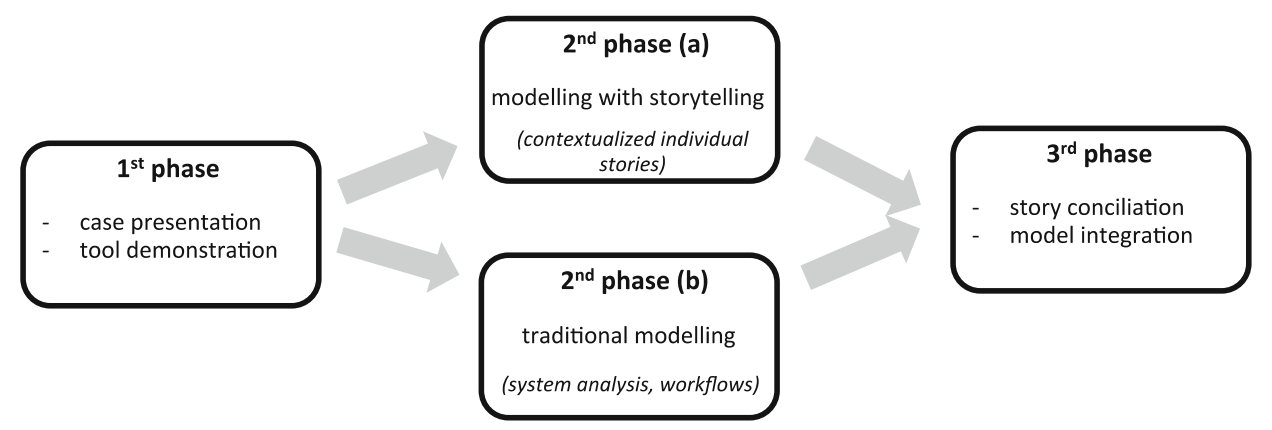

on-site motivational session with all participants, presenting the process story concept and having a quick hands-on demonstration of the tool use, with time for answering questions. This session lasted approximately $1 \mathrm{~h}$.

In the second phase, the participants were split into four groups of three to four persons. On a certain day, a group would be asked to individually use the modeling tool to tell a story about a concrete situation that involved the diploma process. Each session was then finished with a short group discussion about modeling with storytelling. These sessions lasted about $1 \mathrm{~h}$ and $10 \mathrm{~min}$, with about $1 \mathrm{~h}$ dedicated to working with the modeling tool.

This arrangement, having participants working individually in small group sessions, was necessary to allow the organization to keep functioning, while at the same time avoiding low participation levels. These sessions were held during office hours.

The groups were intentionally kept small to facilitate support to users, should any problem arise. When the participants where developing their process stories, we restricted our interventions to resolve technical and usability issues, avoiding any interference on anything related to how they would do it.

As we were interested in learning how participants reacted to the modeling method, we observed them during the sessions. Whenever relevant reactions, comments or dialogues were identified, we silently took notes. This qualitative data is reported together with the case results in the next sections, whenever appropriate.

The participants were allowed to submit more than one story; and they were also allowed to attend more than one session, if necessary to finish their stories. In between sessions, the tool was available on the Internet and participants were encouraged to use it to complete or submit additional stories.

In parallel with the group sessions, we interviewed key staff to gather information about the diploma process. Our aim was to build a reference process model using traditional methods. Besides the interviews, we also witnessed running processes and analyzed data records.
In the third phase of this study, we reconciled the various individual stories and built an integrated process model based on the converged narrative. This model was then analyzed against the reference process model in order to assess on whether the storytelling-based elicitation retained the essential characteristics of the process.

\section{Case Description and Results}

The selected business process concerns obtaining a course certificate for a study cycle, which can either be an undergraduate or a master's course. In both cases, the process begins with a student approaching the academic division's front office (henceforth referred to as FO) with a request for a certificate. The worker attending the student then performs a number of verifications to ensure that all requirements have been met and the certificate can be issued. These include confirming that all course subjects have been completed, the respective marks have been issued, and that all tuition fees have been paid. The student's identity must also be validated and in case the request is being made on the student's behalf, the requester must produce a valid authorization. Once the request is validated, the student is forwarded to the academic division's treasury office (TRE) to proceed with payment. The treasury worker will later on hand over the process to the coordinator, who will distribute it to one of the administrative staff from the back office (BO). Thenceforth, the $\mathrm{BO}$ operative who receives the process checks again the student's academic history, calculates the course average mark, and issues the certificate. The operative then hands over the process to another BO staff member who checks all data for errors and sends the certificate to the Chief of Division, who in turn signs the certificate and sends it to the Dean's office (DO) administrator. The DO administrator signs the certificate and sends it back to the $\mathrm{BO}$, where it is processed and mailed to the student by the same worker that originally handled the request.

As described in the previous section, the data gathering process was organized in three phases, as outlined in 
Fig. 3. The following subsections present the results from each of those stages.

\subsection{First Phase - Motivational Session}

From the initial motivation session we learnt that motivation amongst the fifteen participants was low to moderate, with few subjects expressing high enthusiasm towards this study. A stressful working environment where complaints are common, problems are often difficult to solve, and rules are not strictly defined, explained this. This scenario highlights that the diploma process, as described above, is an oversimplified description of the "happy path" (Correia 2014), thus missing many exceptions and variations.

About one-third of the participants in the motivation session thought the tool would be difficult to use, with the rest of the group remaining cautiously optimistic. Two subjects were particularly apprehensive as they thought they would not be able to use the tool at all.

\subsection{Second Phase - Building Process Stories with the Tool}

Regarding the second phase, we recall that we divided data collection in two parallel streams. We now report on the storytelling sessions (phase 2.a in Fig. 3).

In two of the sessions, some participants revealed resistance in telling concrete details about their working practice, stating that the operating procedure was standard and thus there would not be anything to tell in a story. Even after some debate by the end of the sessions, they generally defended that there were no special cases featuring unexpected circumstances, so the stories would be uninteresting and redundant. However, other participants were more enthusiastic about their stories. This was reinforced by their involvement beyond the assigned sessions, as they were allowed to attend additional sessions and access their stories remotely. In one situation, interesting group behavior emerged in which one participant belonging to $\mathrm{FO}$ was vocally commenting on the FO's viewpoint whilst composing the story with the tool. Our study protocol did not prohibit it, so we considered the phenomenon part of the modeling activity and kept to our observer status. The discussion that was sparkled around the peculiarity of the story led a fellow participant from TRE to submit an additional story to complete the original account with further details from the treasury office. However, interactions amongst the participants did not occur in other sessions.

By the end of phase two, we received a total of twenty individual stories created with the tool. Of these, three stories described other processes in the organization and were thus not considered for all metrics specific to the diploma process.

Every participant was able to compose at least one relevant business story. About half of the participants asked for assistance during the modeling sessions but most of the times to clarify minor usability issues that were immediately addressed. Two participants were unsure how to structure their stories and asked how detailed should each scene be. Owing to our study protocol, we refrained from interfering and stated that any option would be acceptable.

Table 2 summarizes the collected data using the measures defined for our first research question (more detailed data is given in the Appendix, available online via http:// link.springer.com). We are able to report that the participants used narrative and dialogue as the primary means of telling process stories. The total word count (narrative plus dialogue) for the process stories averaged 206 words, with a slight predominance of narrative over dialogue (118 versus 88 ). Standard deviations were moderately high at 122 for the total word count, and 59 and 79 for the narrative and dialogue word counts, signaling ample variations in the use of textual content. Interquartile ranges show a typical variation across stories of 69 words in narrative content and 93 words in dialogue. In both cases, as in the number of scenes, the mean and median values are fairly close and the nonparametric skew is inferior to 0.15 . The only possible outliers are word counts for narrative and dialogue in story 5, (274 and 319, respectively), which rise higher than 1.5 interquartile ranges above the third quartile. Most stories contained a relatively small number of scenes (they averaged 6 scenes per story with a standard deviation of 3), each using a roughly even combination of narrative and dialogue to describe multiple activities in the process. Despite the low number of scenes, many stories featured high scene density, which we defined as the number of identified process activities per scene. Twelve of the process stories included scenes encompassing five or more activities, and one of the stories was completely contained in one scene with 11 activities. Conversely, in some stories, a small subset of scenes did not map to any specific

Table 2 Collected data per story $(\mathrm{n}=20)$ at the end of phase $2 . \mathrm{a}$ (metrics for RQ1)

\begin{tabular}{lllllr}
\hline Variable & M & SD & Q1 & Mdn & Q3 \\
\hline Number of scenes & 5.7 & 3.0 & 3 & 5.5 & 7 \\
Word count (narrative) & 118.3 & 58.9 & 82 & 115 & 151 \\
Word count (dialogue) & 87.7 & 78.9 & 26.5 & 76 & 119 \\
Variable & Low & Medium & High & & \\
Structural complexity & 5 & 8 & 7 & & \\
\hline
\end{tabular}


activities and were used for setting the context or detailing findings (stories 11,20), or explaining thought processes (stories 2, 13, 14). These results are also relevant for RQ3 and are further detailed in Sect. 4.4 with the results from phase 3.

None of the participants used explicit control mechanisms to structure their stories, relying instead on textual descriptions provided in scene metadata. These results support the view that the participants described actual procedures in concrete, real situations, rather than general views or idealized procedures. It also supports the view that, unlike participants with background in computer science (as in our second set of experiments), business people seem to be less focused on coordination. In short, the participants told process stories according to their specific work context, not according to the business process as a whole. We observed that the complete process could only emerge from the reconciliation of several stories in phase 3 . We based our qualitative assessment regarding structural complexity on how many actors intervened in the story, and the number of decisions contained in the identified activities impacting process flow. For instance, story 3, which rated medium in structural complexity, featured three actors and described two relevant process decisions. All recorded stories verified the defined process validity requirements.

Regarding the metrics for RQ2, we now analyze the depiction of unexpected situations and the expression of personal views in the stories. Detachment from prescribed procedure will be analyzed together with the results from phase 3. We found that fifteen of the twenty stories described unexpected situations, which shows that participants, when recounting concrete events in their work routines, were more prone to choose and highlight atypical situations, detailing the circumstances that led to unanticipated scenarios, and exposing the contextual reasoning that steered their actions.

We found that eight of the stories included personal views in the narrative or dialogue, signaled by emotional elements such as anger, disbelief, empathy, or condescendence. For instance, in one story the parents of a student try to obtain their son's course certificate, but the FO operative discovers that the student is missing several courses and is still attending the first year. Unable to give out such information to the parents, the operative consults the BO coordinator and upon return denotes that the request cannot be accepted because "not all grades have been issued" (directly cited from the story's narrative). As shown in Fig. 4 (left), the operative tries to empathize with the parent, saying that it would be "easier" if he gets a power of attorney. The narrator signals the operative's discomfort, indicating that the operative was "trying to avoid an awkward situation". The parent was however not satisfied and "contested the necessity for a power of attorney". The irritation behind the parent's reply in scene 3 further builds in the following scene (not shown in the figure for lack of space): in the dialogue, the operative states, "The student is of age", to which the parent retorts: "Can you tell us our son's situation?" The parent's indignation is confirmed in scene 5 (shown right in the figure).

In Fig. 5 we show another example of a scene bearing emotional traits that expose personal views from the actors. In this instance, an aggravated student repeatedly insists on being sent both a course certificate and a course diploma, even though the existing procedures dictate that course diplomas are handed separately, on a public ceremony. After some debate with the attending operative, the operative reluctantly concedes to the student's request and works with the back-office colleagues to accommodate this special request. The narrative passages associated with this particular story and the titles given to the scenes complement the emotional elements in the dialogue by framing them in the developing storyline, and underline the reasons behind the student's anger. Among the narrator's remarks, we can read: "The student was unaware that he would not be taking the diploma", and "The student insists that he needs the diploma because he is going to work abroad".

\subsection{Second Phase B - Traditional Modeling}

Parallel to the individual storytelling sessions, in phase 2 we also interviewed the Chief of Division, the back-office coordinator and an experienced member of staff to model the standard diploma process using conventional techniques (phase 2.b in Fig. 3). We found out that there were no written procedures for the diploma process. We therefore put together the process model from interviews, observation and document analysis. The Chief of Division offered a broad, top-level report of the happy process and even showed an application used to monitor the diploma processes being handled by the academic division. The Back-Office coordinator and staff member provided more thorough information, detailing each step in the process and describing the specific requirements for accepting requests, verification lists, service options and pricing, and prescribed procedures across multiple scenarios. These descriptions were in accordance with the more general account provided by the Chief of Division and were remarkably similar, despite being highly detailed. Further analysis of these results and especially the reference process model created in this stage, will be presented in tandem with the discussion of phase three. 
Fig. 4 Excerpts from a story exposing personal views (story 9, scenes 3 and 5, translated to English)
Fig. 5 Excerpt of a story conveying emotion (story 18 , scene 8, translated to English)
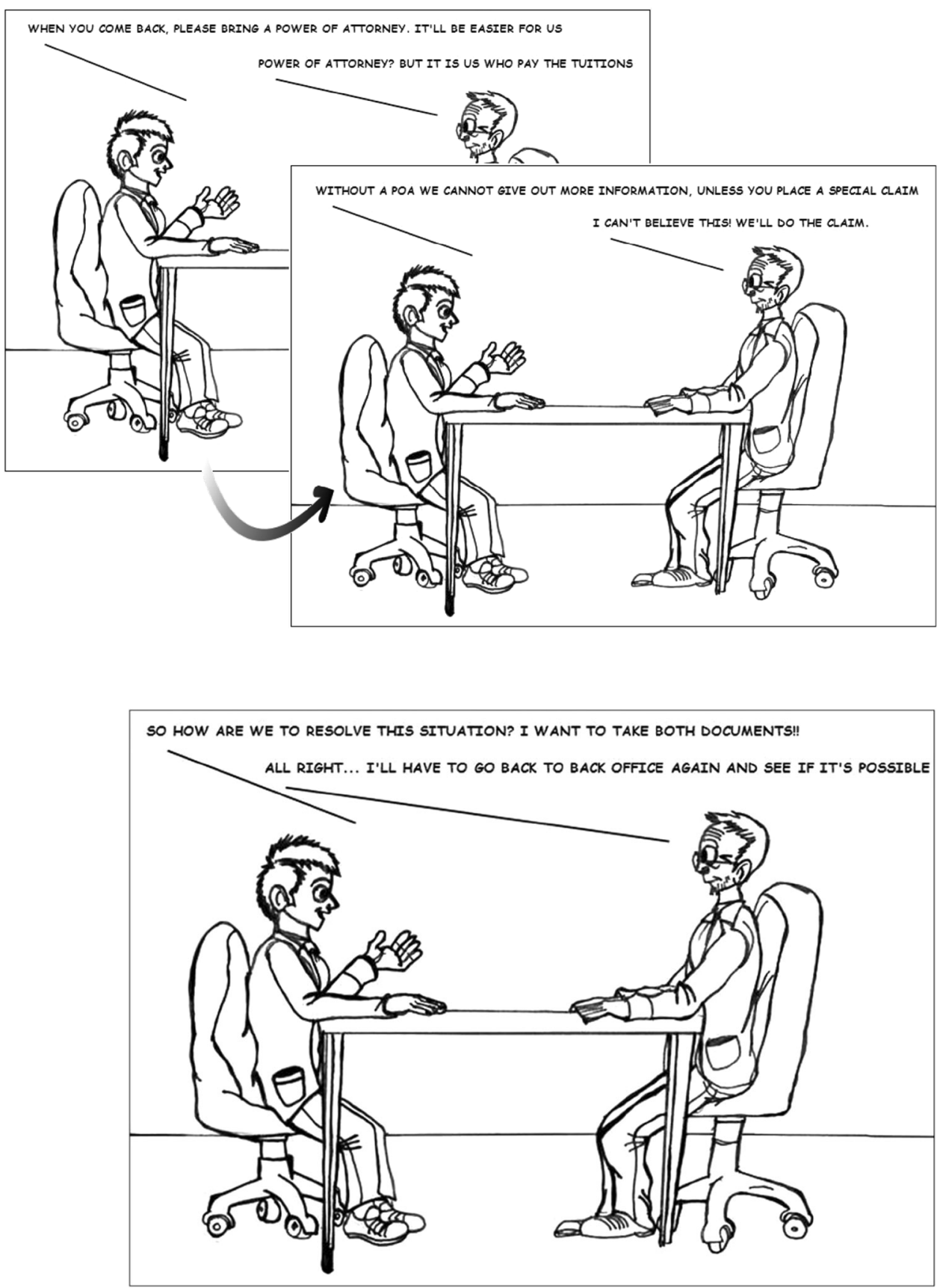

\subsection{Third Phase - Story Conciliation and Model} Integration

In the third phase we conducted a thorough analysis of the process stories and combined them into a unified, generalized process story. We divided the individual stories into three segments, according to the type of content that was processed: (1) student attending (ATT), covering all activities related to dealing directly with the student and checking prerequisites; (2) payment handling (PAY); and (3) request processing (PRO), wrapping all activities leading to issuing a certificate and delivering it to the student.

We found out that the participants dedicated varied levels of attention to these story segments, giving ample "story time" to the description of activities that were directly tied to their work responsibilities, while deemphasizing or completely ignoring other parts of the process. The level of detail given to each segment followed the same trend - as it is shown in Fig. 6, the level of detail across segments was heterogeneous in all stories, with participants from the $\mathrm{FO}$ and $\mathrm{BO}$ groups giving more 
Fig. 6 Level of detail for each segment in the stories. Stacked blocks show the coverage of process segments across individual stories. (Stories 4, 15, 20 were not considered because they did not describe the diploma process)

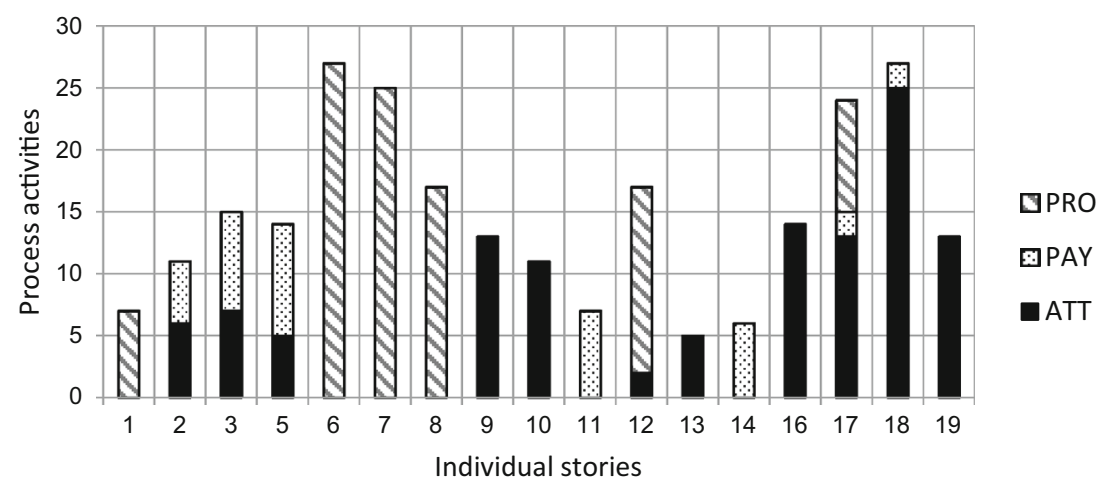

detailed accounts of the ATT and PRO segments, and subjects from treasury providing more thorough descriptions of the corresponding segment in their stories. In story 17 , we could observe highly detailed explanations in both the ATT and PRO segments, which was explained when we found that the author of story 17 collaborated closely with both groups (that particular story was created by the BO coordinator). None of the stories that focus on the PAY segment provided detailed accounts of the remaining parts of the process, due to the relatively independent role of the treasury group within the academic division (all stories were created by subjects from TRE). As we will see subsequently, we were able to combine elements from all stories to obtain a balanced, highly detailed converged description across the three story segments.

To produce an integrated model from the various individual stories, we first created a BPMN model for each individual story. Due to space constraints, these models are not shown here. Instead, in the Online Appendix we provide one of the BPMN models as an example and some quantitative data on all models.

To the best of our knowledge, there is no proposed methodology to transform and integrate scene-based process stories into standard process models. Our approach is similar to the technique proposed by Kabicher and Rinderle-Ma (2011), built on content analysis from written process descriptions and aggregation of elicited process activities. Based on a preliminary analysis of the stories, we identified the three story segments outlined above. We then classified groups of scenes into each story segment and analyzed narrative and dialogue content, actors, artefacts, and other metadata in each scene in order to identify key process elements - activities, roles, decisions, and events. We then used these elements together with the structure embedded in the story and the sequencing of scenes to form one process model per story.

By comparing the number of activities in the synthesized models with the number of scenes in individual process stories, we can conclude that scenes do not map directly to activities. Most process stories display high scene density, with each scene encompassing multiple activities (six to seven in some cases). At the other end of the spectrum, there are scenes that do not translate to any activity, as they are used to describe the context, explain findings, or depicting interactions through dialogue. Looking at the breakdown of activities per story segment, we found out that stories were typically focused on specific parts of the procedure, which is consistent with the fact that the participants were describing concrete scenarios that happened in their daily routine. All but one story ignored at least one of the segments in the complete procedure, and ten stories were completely dedicated to a particular segment. The standard process elicited in phase 2.b through traditional process modeling techniques was in average more detailed than the stories in all segments (if we discard the stories that did not address each segment, it was still above average in the ATT and PRO segments), but was surpassed by individual accounts in all segments. However, the standard process embodied more decision steps than any of the individual accounts.

Interestingly, all individual stories included situations or details that were not present in the standard process. For instance, story 13 depicts a case where a student requests a duplicate course certificate due to a name change, a situation that the standard process does not predict. In contrast with the more high-level description in the standard process, stories 2 and 16-19 provide ample details regarding verifying a students' application for a course certificate, which cover different situations where thorough analysis is required, as well as various steps necessary to overcome typical problems with the students' applications.

Furthermore, six of the stories contained elements that went as far as to contradict prescribed procedure. For instance, in stories 17 and 18 students are allowed to request a course certificate even though the services cannot verify that all courses have been completed. In both stories, the attending operatives ask a student to provide a signed declaration acknowledging that the certificate would only be issued upon confirmation that all course marks had been issued. Story 18 deviates again from the standard process 


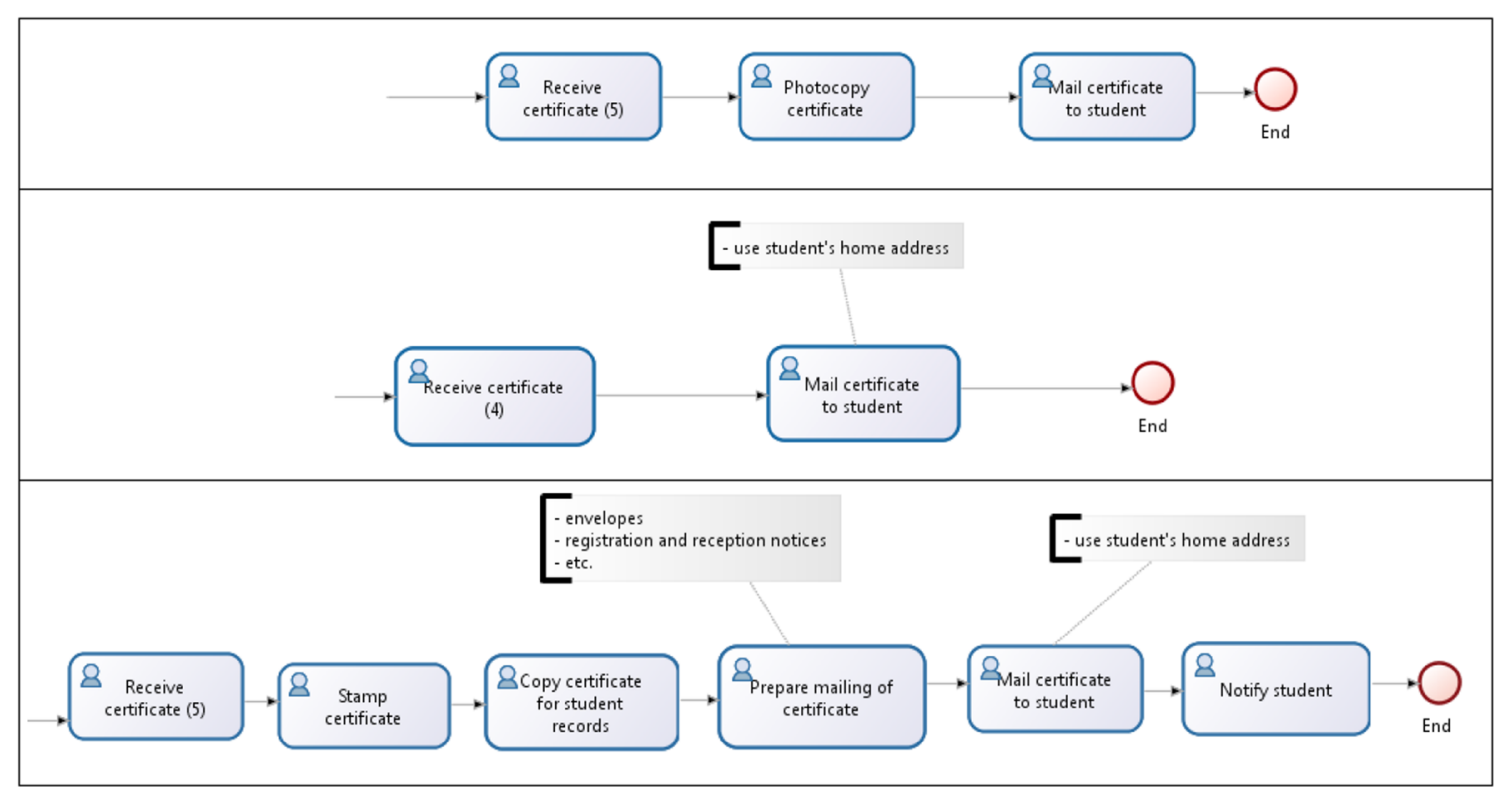

Fig. 7 Combining activity streams into a single sequence. Activities from story 6 are displayed top-left, story 7 is top-right, and at the bottom is the converged model (produced from scenes from two additional stories)

when a student insists on being mailed both the course certificate and the course diploma (recall the scene illustrated in Fig. 5).

Once all individual process stories were converted to process models, we integrated the various models into a converged process model with information from all stories. We handled different labelling of activities and artefacts by identifying common elements in overlapping stories, analyzing their frequency of occurrence and adopting inductive elaboration of labels (derive labels from text and generalize). We handled variations in granularity in two steps. The first step, summarization, was performed when converting each process story into a process model. Neutral or decorative textual elements were removed and a summarized activity description was produced from dialogue passages and narrative content in the scene. The second step, aggregation, took place during model integration. Its objective was to combine overlapping activities while preserving detail. Multiple, complementary accounts of a procedure were unified into a unique sequence of activities (as shown in Fig. 7), while at the same time preserving variations using exclusive gateways (as exemplified in Fig. 8).

The final converged process model contained 125 nodes including 34 decisions. It incorporated individual contributions from 13 participants divided between different organizational roles. Table 3 shows measurements from story models (averaged), the prescribed procedure, and the converged model, using metrics from Kunze et al. (2011) and the BPM Academic Initiative (BPMAI ${ }^{1}$ ) process collection (averaged) as a baseline. We use the number of nodes $(N N)$ as the size metric, and three metrics for density: the coefficient of connectivity $(C N C)$ as the ratio between edges and nodes, and the average and maximum degree of routing ( $A v g D R$ and MaxDR) as the average and maximum number of nodes a routing node is connected to.

These measurements show that the size of the diploma process (prescribed procedure) is considerable, containing more nodes than the BPMAI models on average. Observed values for all density metrics are also higher on average. Measurements from individual story models are comparable on average to BPMAI models. These measurements also indicate that the converged model provided a more detailed, richer representation of the diploma process, with $35 \%$ higher $C N C$ and $229 \%$ increase in the number of process nodes when compared to the prescribed procedure. Nonetheless, we note that there are some details that can only be found in the standard process, suggesting that a combination of traditional and storytelling may yield the best results. Specifically, the converged model does not describe the scenario whereby a student requests to pick up the certificate in person. It presents the mailing of the certificate to the student's home address as the only option (recall Fig. 7), in contrast with the standard process, which contemplates both scenarios. Apart from this subtlety, the converged model covers and surpasses in detail every other aspect of the standard process. This suggests that a relatively small number of process stories may be sufficient to model a business process.

\footnotetext{
${ }^{1}$ http://bpt.hpi.uni-potsdam.de/BPMAcademicInitiative.
} 
Table 3 Measurements from individual process models (avgd), the BPMAI model collection (avgd), prescribed procedure, and the final converged model

\begin{tabular}{lcllc}
\hline Model & Size $N N$ & Density $C N C$ & $\begin{array}{l}\text { Density } \\
\text { AvgDR }\end{array}$ & Density MaxDR \\
\hline Story models (Avg) & 14.0 & 0.92 & 1.12 & 1.21 \\
BPMAI (Avg) & 15.6 & 0.79 & 1.15 & 1.84 \\
Prescribed procedure & 38 & 1.08 & 1.67 & 2.0 \\
Converged model & 125 & 1.46 & 1.56 & 3.0 \\
\hline
\end{tabular}

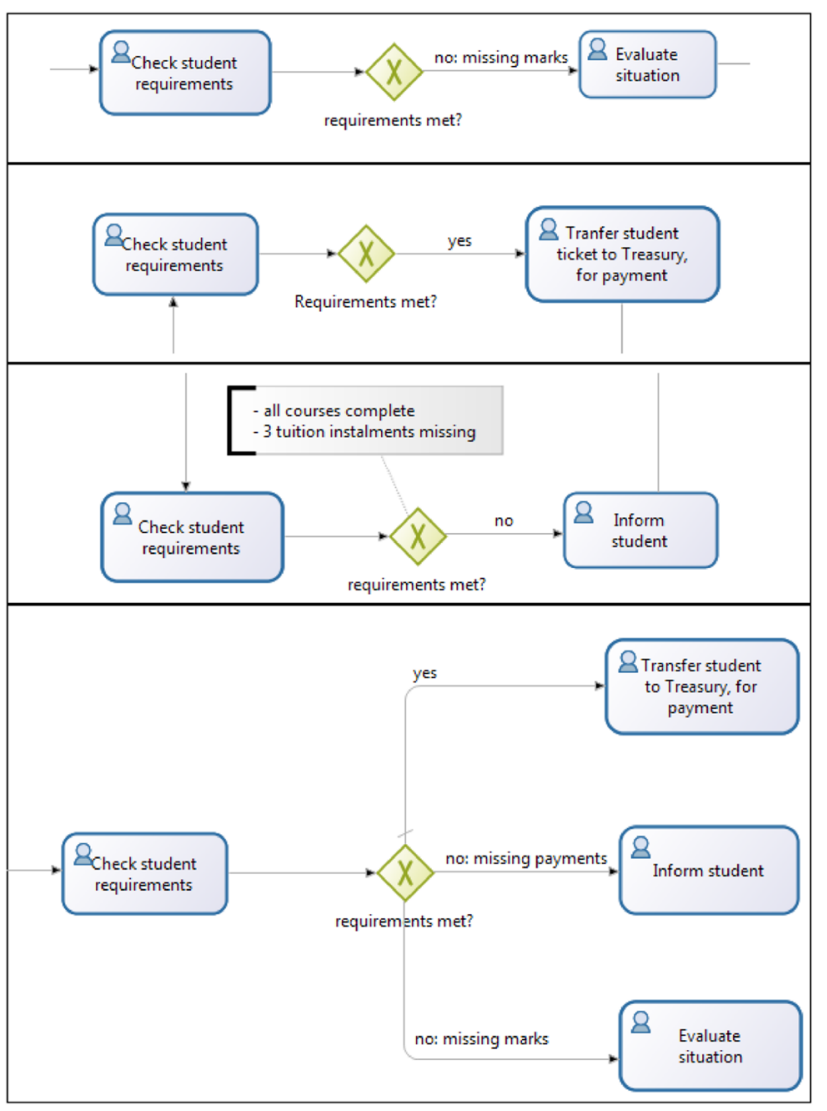

Fig. 8 Combining alternative scenarios with an exclusive (XOR) gateway. From top to bottom: activities from story 2, 3 and 10, converged model. The excerpt from the converged model was simplified for clarity

The analysis of some process stories also suggests that iterating over time instead of capturing stories in parallel may avoid missing process details. For instance, the converged model describes the procedure taken when missing tuition payments are detected by the administrators. If all verifications fail (details not given here for brevity), the student is given the opportunity to present an ATM receipt and only after failing to do so is the certificate request denied. While this is considerably more detailed than the standard process, which only states that requests with missing payments should be denied, the story reveals that there are details that remain unknown. For instance, it is unclear what conciliating steps would have been taken if the student had produced the requested receipts. The gathering of additional process stories over time could capture such scenario.

\subsection{Limitations}

This study does not seek to provide a direct comparison between storytelling and traditional modeling techniques. Although during the traditional modeling phase we carefully observed procedures and analyzed process documents, there were fewer participants involved in building the reference model. Therefore, it may be argued, it should be expected that there would be less elicited process activities. We acknowledge that limitation, but we note that stories gathered from subjects participating in both stages offered more detail than those offered during interviews. Additionally, we point out that the participants who were involved in establishing the reference model were precisely the ones responsible for defining the diploma process (Chief of Division) and implementing the prescribed procedure (Back-Office Coordinator and the experienced staff member). The fact that we were seeking to elicit the prescribed procedure was the main reason why we restricted this phase to key personnel.

External threats to the study's validity are also acknowledged, specifically the extent to which we can use our findings in the present study towards building a generalized approach to process modeling. We addressed this problem by identifying issues in previous studies that could impact the generalization of our results. Using a different organization from previous studies contributes to mitigate biases, even though we recognize that a cross case analysis would have been beneficial.

\section{Discussion}

We now evaluate the results from the case study by reconsidering our research questions. 
5.1 Is it Feasible for Stakeholders to Elicit Meaningful Business Processes Without the Participation of Expert Modelers?

The data we have collected clearly shows that stakeholders can indeed autonomously model their own work activities. Out of the twenty stories that were documented with the tool, seventeen depicted valid use case scenarios of the target process (two stories described other processes in the organization and one story could not be mapped to a process description). Unlike our previous studies, the participants were able and keen to create business process stories whilst fully adhering to the approach. Despite being composed of relatively small sets of sequential scenes (ranging from 1 to 12), the generated process stories relied heavily on dialogue and textual descriptions to convey the storyline. Individual stories averaged a combined total of 206 words between dialogue and narrative text, each depicting up to 27 process activities in between up to 6 organizational roles. As it was covered in the analysis, we were able to translate these stories into a unified BPMN business process model.

\subsection{Is Business Process Context Extractable from Process Stories?}

Business process context cannot be fully understood by analyzing prescribed procedure alone. Part of that context stems from varying circumstances across process instances. As they are dealt with, workers become more experienced but this knowledge remains tacit in their minds and it is difficult if at all possible to articulate (Polanyi 1967; Baddeley 1992). Although we cannot assert that this tacit knowledge is being externalized in process stories, they do contain elements showing that context information is being preserved as a form of situated recall, from which tacit knowledge can be more easily primed and shared (Brown and Duguid 1998; Bennet and Bennet 2008). They are (1) the presence of emotional elements within the narrative in eight of the stories, signaling an emotional engagement of the author and transmitting information often untapped by traditional elicitation techniques, such as the role of emotion (e.g. irritation, empathy) on making decisions impacting process flow; (2) the depiction of unexpected situations in almost all of the stories; with (3) corresponding contextualized descriptions explaining the actors' reasoning and detailing their responses towards resolving those situations. The occurrence of the latter two items in the stories constituted a documented contextualized account on the behavior of experienced workers when facing real-world situations in their daily routines. These behaviours, grounded on implicit knowledge stemming from years of experience, often deviated from approved practices and at times went as far as contradicting them, although preserving the intended outcome of the prescribed procedure.

\subsection{How do Process Stories Improve the Modeling of Business Processes?}

Our results indicate that stakeholder-centered storytelling is a valuable technique towards improving process elicitation and modeling. In this particular study, the creation of individual process stories by staff unveiled numerous de facto practices that were not captured by traditional process elicitation and modeling. These practices, while not featured in the standard process, are common in the work routine, and appeared in multiple stories with small variations and adaptations according to context. By analyzing the different process stories we could observe that the operatives' activities were supported on a mental model of the process (versus an opaque rigid set of operating rules) and were driven by intention. For instance, the several stories that workers chose to externalize suggest workers understand the particular reasons behind the students' unusual requirements when requesting diplomas. By collaborating with various units within the organization and exerting contextualized reasoning, they seem to be in some circumstances able to tackle situations where prerequisites are not met, and still provide the service to the student. The fact that these operational practices were captured in the process stories shows that our method was successful in modeling the actual process versus an idealized version based on an abstract rule-based workflow.

By combining different process scenarios detailed in individual stakeholder accounts, we were able to compose a highly detailed process model based on actual procedure, which constitutes a valuable artefact for evaluating and enriching the reference model. Finally, we note the converged model was presented to the Chief of Division, who apart from minor discrepancies validated it as satisfying the standard process. The reference model had already been validated at the end of phase 2.b. The organization is currently undergoing steps to document its processes with the aim of meeting quality requirements and automating procedures for increased control and efficiency.

\section{Conclusions and Future Work}

This paper presented a new approach for process modeling centered on the elicitation and analysis of process stories composed by stakeholders. A benefit of this approach relies with how it balances descriptive, creative aspects of 
storytelling with constrained, activity-oriented aspects of process modeling. The storytelling environment steers the stakeholders into creating concrete, event-based process stories, while at the same time granting space for them to supply stories with contextual explanations and subjective remarks. The results from our case study have confirmed that stakeholders without IT backgrounds and modeling expertise are able to create process stories using our method. Moreover, they show that the storytelling method applied to process modeling is an effective technique for eliciting process stories containing detailed contextualized accounts of use case scenarios that have actually taken place in the work routine and that can deviate or even contradict prescribed procedures. These process stories are descriptive but grounded on the sequencing of process activities, and thus can be translated and integrated into traditional process models. This approach can play a complementing role to traditional modeling techniques by capturing process knowledge that is often implicit and difficult to transmit by other means. Process stories constitute an asset for retaining organizational knowledge and sparkling discussion in modeling and reengineering teams.

These research results open up some interesting avenues for future research. A promising research line concerns theory building, which may depart from the positive indications provided by the adoption of storytelling and sensemaking theories to further develop a theory about business process contextualization, process richness, flexibility, and human behavior. In turn, such theory could lead towards the development of process modeling languages more centered on human and organizational needs rather than on technical ones.

Another research line to consider brings back the various criteria and specific measures we adopted in this study to analyze process stories, assessing in particular the structural complexity, expression of personal views, depiction of unexpected situations, and number of decision steps, among others. Further research could explore the value of these criteria to assess model quality.

The very positive signs brought about by this study suggesting that stakeholders can generate process models, even though some participants in the study expressed initial concerns that they would be unable to do so, advise further research on how to increase the usability of existing process modeling languages and tools, and also how to increase collaboration in process elicitation and modeling. From a wider perspective, we note an interesting trend moving process modeling from modeling experts to stakeholders. Another interesting possibility would be crowd-sourcing process modeling, which may allow large organizations to reduce the costs of BPM initiatives while increasing agility and maintainability.

\section{References}

Aguilar-Saven RS (2004) Business process modelling: review and framework. Int J Prod Econ 90:129-149. doi:10.1016/S09255273(03)00102-6

Antunes P, Mourão H (2011) Resilient business process management: framework and services. Expert Syst Appl 38:1241-1254. doi:10.1016/j.eswa.2010.05.017

Antunes P, Simões D, Carriço L, Pino JA (2013) An end-user approach to business process modeling. J Netw Comput Appl 36:1466-1479. doi:10.1016/j.jnca.2013.03.014

Baddeley A (1992) Working memory. Science 255:556-559

Bandara W, Gable GG, Rosemann M (2005) Factors and measures of business process modelling: model building through a multiple case study. Eur J Inf Syst 14:347-360

Basili VR, Weiss DM (1984) A methodology for collecting valid software engineering data. IEEE Trans Softw Eng (TSE) 10:728-738. doi:10.1109/TSE.1984.5010301

Benbasat I, Goldstein DK, Mead M (1987) The case research strategy in studies of information systems. MIS Q 11(3):369-386

Bennet D, Bennet A (2008) Engaging tacit knowledge in support of organizational learning. Vine 38:72-94. doi:10.1108/ 03055720810870905

Borges MRS, Pino JA (1999) PAWS: towards a participatory approach to business process reengineering. In: String processing and information retrieval symposium, 1999 and international workshop on groupware (SPIRE/CRIWG). IEEE Comput. Soc, Cancun, pp 262-269

Brossard A, Abed M, Kolski C (2011) Taking context into account in conceptual models using a model driven engineering approach. Inf Softw Technol 53:1349-1369. doi:10.1016/j.infsof.2011.06. 011

Brown JS, Duguid P (1998) Organizing knowledge. Calif Manag Rev 40:90-111

Cabitza F, Simone C (2013) Computational coordination mechanisms: a tale of a struggle for flexibility. Comput Support Coop Work (CSCW) 22:475-529. doi:10.1007/s10606-013-9187-5

Caetano A, Silva AR, Tribolet J (2005) Using roles and business objects to model and understand business processes. In: Proceedings of the ACM symposium on applied computing (SAC '05). ACM, Santa Fe, pp 1308-1313

Chakraborty S, Sarker S, Sarker S (2010) An exploration into the process of requirements elicitation: a grounded approach. J Assoc Inf Syst 11:212-249

Correia ACE (2014) Quality of process modeling using BPMN: a model-driven approach. Dissertation, Universidade Nova de Lisboa

da Cunha Mattos T, Santoro FM, Revoredo K, Nunes VT (2014) A formal representation for context-aware business processes. Comput Ind 65:1193-1214. doi:10.1016/j.compind.2014.07.005

Denning S (2000) The springboard: how storytelling ignites action in knowledge-era organizations. J Organ Change Manag 14:609-614. doi:10.1108/jocm.2001.14.6.609.2

Denning S (2006) Effective storytelling: strategic business narrative techniques. Strateg Leadersh 34:42-48. doi:10.1108/ 10878570610637885

Dumas M, La Rosa M, Mendling J, Reijers HA (2013) Fundamentals of business process management. Springer, New York. doi:10. 1007/978-3-642-33143-5

Figl K, Recker J (2014) Exploring cognitive style and task-specific preferences for process representations. Requir Eng 19:1-23. doi:10.1007/s00766-014-0210-2

Fleischmann A, Schmidt W, Stary C, Obermeier S, Brger E (2012) Subject-oriented business process management. Springer, Heidelberg, New York. doi:10.1007/978-3-642-32392-8 
Fleischmann A, Kannengiesser U, Schmidt W, Stary C (2013) Subject-oriented modeling and execution of multi-agent business processes. In: 2013 IEEE/WIC/ACM international joint conferences on web intelligence (WI) and intelligent agent technologies (IAT), vol 2. IEEE, pp 138-145

Gershon N, Page W (2001) What storytelling can do for information visualization. Commun ACM 44:31-37

Giaglis GM (2001) A taxonomy of business process modeling and information systems modeling techniques. Int J Flex Manuf Syst 13:209-228. doi:10.1023/A:1011139719773

Harman J, Brown R, Johnson D, Rinderle-Ma S, Kannengiesser U (2015) Virtual business role-play: leveraging familiar environments to prime stakeholder memory during process elicitation. In: Zdravkovic J, Kirikova M, Johannesson P (eds) Advanced information systems engineering. CAiSE 2015. Lecture Notes in Computer Science, vol 9097. Springer, Cham, pp 166-180

Holt AW, Ramsey HR, Grimes JD (1983) Coordination system technology as the basis for a programming environment. Electr Commun 57:307-314

Hommes BJ, van Reijswoud V (2000) Assessing the quality of business process modelling techniques. In: Proceedings of the 33rd annual Hawaii international conference on system sciences, vol 1. Maui, p 1007

Jonnavithula L, Antunes P, Cranefield J, Pino JA (2015) Organisational issues in modelling business processes: an activity-based inventory and directions for research. In: 19th Pacific Asia conference on information systems (PACIS), Singapore

Kabicher S, Rinderle-Ma S (2011) Human-centered process engineering based on content analysis and process view aggregation. In: Mouratidis $\mathrm{H}$, Rolland $\mathrm{C}$ (eds) Advanced information systems engineering. CAiSE 2011. Lecture Notes in Computer Science, vol 6741. Springer, Berlin, Heidelberg. doi: 10.1007/ 978-3-642-21640-4

Kock N, Verville J, Danesh-Pajou A, DeLuca D (2009) Communication flow orientation in business process modeling and its effect on redesign success: results from a field study. Decis Support Syst 46:562-575. doi:10.1016/j.dss.2008.10.002

Krogstie J (2007) Modelling of the people, by the people, for the people. In: Krogstie J, Opdahl AL, Brinkkemper S (eds) Conceptual modelling in information systems engineering. Springer, Berlin, Heidelberg, pp 305-318. doi:10.1007/978-3540-72677-7_19

Kunze M, Luebbe A, Weidlich M, Weske M (2011) Towards understanding process modeling - the case of the BPM academic initiative. Business process model and notation, vol 95. Springer, Heidelberg, pp 44-58

Lakin R, Capon N, Botten N (1996) BPR enabling software for the financial services industry. Manag Serv 40(3):18-21

Leopold H, Mendling J, Polyvyanyy A (2014) Supporting process model validation through natural language generation. IEEE Trans Softw Eng 40:818-840. doi:10.1109/TSE.2014.2327044

Lethbridge TC, Sim SE, Singer J (2005) Studying software engineers: data collection techniques for software field studies. Empir Softw Eng 10:311-341. doi:10.1007/s10664-005-1290-x

Lindsay A, Downs D, Lunn K (2003) Business processes-attempts to find a definition. Inf Softw Technol 45:1015-1019. doi:10. 1016/S0950-5849(03)00129-0

List B, Korherr B (2006) An evaluation of conceptual business process modelling languages. In: Proceedings of the 2006 ACM symposium on Applied computing (SAC '06). ACM, New York, pp 1532-1539

Mayer RE (1989) Models for understanding. Rev Educ Res 59:43-64. doi:10.3102/00346543059001043

Mayer RJ, Menzel CP, Painter MK, Dewitte PS, Blinn T (1995) Information integration for concurrent engineering (IICE),
IDEF3 process description capture method report. http://www. idef.com/pdf/idef3_fn.pdf. Accessed 30 Mar 2015

McCloud S (1993) Understanding comics: the invisible art. William Morrow Paperbacks, Northampton

Mendling J, Recker J, Reijers HA (2010) On the usage of labels and icons in business process modeling. Int J Inf Syst Model Des (IJISMD) 1:40-58. doi:10.4018/jismd.2010040103

Nardella K, Brown RA, Kriglstein S (2014) Storyboard augmentation of process model grammars for stakeholder communication. In: International conference on information visualization theory and applications. SCITEPRESS-Science and Technology Publications, pp 114-121

Object Management Group (2011) Unified modelling language (OMG UML) superstructure. http://www.omg.org/spec/UML/2. 4.1/Superstructure. Accessed 15 Jun 2015

Object Management Group (2013) Business process model and notation (BPMN). http://www.omg.org/spec/BPMN/2.0.2/PDF. Accessed 15 Jun 2015

Ottensooser A, Fekete A, Reijers HA, Mendling J, Menictas C (2012) Making sense of business process descriptions: an experimental comparison of graphical and textual notations. J Syst Softw 85:596-606. doi:10.1016/j.jss.2011.09.023

Perret R, Borges MRS, Santoro FM (2004) Applying group storytelling in knowledge management. In: de Vreede GJ, Guerrero LA, Marín Raventós G (eds) Groupware: design, implementation, and use. CRIWG 2004. Lecture Notes in Computer Science, vol 3198. Springer, Berlin, Heidelberg, pp 34-41

Pesic M (2008) Constraint-based workflow management systems: shifting control to users. Dissertation, Technische Universiteit Eindhoven

Polanyi M (1967) The tacit dimension. Anchor, Garden City

Recker JC, Dreiling A (2007) Does it matter which process modelling language we teach or use? An experimental study on understanding process modelling languages without formal education. ACIS. University of Southern Queensland, Toowoomba, pp 356-366

Recker J, Indulska M, Rosemann M, Green PF (2006) How good is BPMN really? Insights from theory and practice. In: 14th European conference on information systems. Goeteborg, pp 1582-1593

Recker J, Safrudin N, Rosemann M (2010) How novices model business processes. In: International conference on business process management. Springer, Heidelberg, pp 29-44

Riemer K, Johnston R, Indulska M (2014) Questioning the philosophical foundations of business process modelling. In: Information systems foundations: theorising in a dynamic discipline. ANU E Press, Canberra, pp 1-16

Rosemann M, Recker J, Flender C (2008) Contextualisation of business processes. Int J Bus Process Integr Manag 3:47-60. doi:10.1504/IJBPIM.2008.019347

Runeson P, Höst M (2009) Guidelines for conducting and reporting case study research in software engineering. Empir Softw Eng 14:131-164

Russell N, van der Aalst W, ter Hofstede A, Wohed P (2006) On the suitability of UML 2.0 activity diagrams for business process modelling. In: Proceedings of the 3rd Asia-Pacific conference on conceptual modelling (APCCM '06), vol 53. Australian Computer Society, Darlinghurst, pp 95-104

Santoro F, Borges M, Pino J (2010) Acquiring knowledge on business processes from stakeholders' stories. Adv Eng Inform 24:138-148. doi:10.1016/j.aei.2009.07.002

Scheer A-W, Thomas O, Adam O (2005) Process modeling using event-driven process chains. Process Aware Inf Syst. doi:10. 1002/0471741442.ch6

Schnupp WRP, Muchnick SS (1992) Primer in petri net design. Springer, Secaucus 
Short JC, Reeves TC (2009) The graphic novel: a "cool” format for communicating to generation Y. Bus Commun Q 72:414-430. doi: $10.1177 / 1080569909336464$

Simões D, Antunes P, Cranefield J (2016) Enriching knowledge in business process modelling: a storytelling approach. In: Razmerita L, Phillips-Wren G, Jain L (eds) Innovations in knowledge management: the impact of social media, semantic web and cloud computing, vol 95. Springer, Heidelberg, pp 241-267. doi:10.1007/978-3-662-47827-1_10

van der Aa H, Leopold H, Mannhardt F, Reijers HA (2015a) On the fragmentation of process information: challenges, solutions, and outlook. Bus process model not, vol 214. Springer, Cham, pp 3-18

van der Aa H, Leopold H, Reijers HA (2015b) Detecting inconsistencies between process models and textual descriptions. In: Motahari-Nezhad H, Recker J, Weidlich M (eds) Business process management. BPM 2015. Lecture Notes in Computer Science, vol 9253. Springer, Cham, pp 90-105. doi: 10.1007/ 978-3-319-23063-4

van der Aalst W (2013) Business process management: a comprehensive survey. ISRN Softw Eng 2013:1-37. doi:10.1155/2013/ 507984 van der Aalst W, ter Hofstede A (2005) YAWL: yet another workflow language. Inf Syst 30:245-275

van der Aalst W, ter Hofstede A, Kiepuszewski B, Barros A (2003) Workflow patterns. Distrib Parallel Databases 14:5-51. doi:10. 1023/A:1022883727209

Weick KE (1993) The collapse of sensemaking in organizations: the Mann Gulch disaster. Adm Sci Q 38:628-652. doi:10.2307/ 2393339

Weick KE (1995) Sensemaking in organizations. Sage, Thousand Oaks

Williams AM, Alspaugh TA (2008) Articulating software requirements comic book style. In: Third international workshop on multimedia and enjoyable requirements engineering-beyond mere descriptions and with more fun and games, pp 4-8

Wohed P, van der Aalst WMP, Dumas M, ter Hofstede AHM, Russell $\mathrm{N}$ (2006) On the suitability of BPMN for business process modelling. Business Process Management, vol 4102. Springer, Heidelberg, pp 161-176 